\title{
Surgical Management of Cervical Vertebral Fractures in Two Dogs with Severe Concurrent Vertebral Venous Plexus Hemorrhage
}

\author{
James W. Guthrie ${ }^{1}$ Noel Fitzpatrick ${ }^{1}$ \\ ${ }^{1}$ Fitzpatrick Referrals, Eashing, Surrey, United Kingdom \\ VCOT Open 2018;1:e1-e7.
}

\begin{abstract}
Address for correspondence James W. Guthrie, BVM\&S, CertAVP (GSAS), DipECVS, MRCVS, Fitzpatrick Referrals, Halfway Lane, Eashing, Surrey GU7 2QQ, United Kingdom (e-mail: jamesg@fitzpatrickreferrals.co.uk).
\end{abstract}

\begin{abstract}
Surgical repair of fractures and luxations of the cranial cervical vertebrae can be complicated by vertebral venous plexus hemorrhage following fracture fragment manipulation and realignment. Severe hemorrhage is potentially life threatening and may preclude definitive surgical fixation if it cannot be acceptably controlled and interferes with fixation technique. This report describes this scenario and a surgical strategy in two dogs with fractures of the second cervical vertebra. The surgical technique to limit hemorrhage included the insertion of the pins into the vertebrae, placement of collagen fleece, and autogenous cancellous bone graft over the fracture site, and while the polymethylmethacrylate cement was still within the liquid phase,

Keywords

- cervical spine fracture

- vertebral venous plexus

- axial distraction axial traction was employed. The long-term outcome was considered excellent with both dogs able to ambulate unassisted and free from signs of pain. Although it is uncommon, severe vertebral venous plexus hemorrhage can occur during cervical vertebrae fracture repair. This technique provides a means to achieve internal fixation while concurrently limiting hemorrhage.
\end{abstract}

\section{Introduction}

Cervical fractures and luxations in dogs are diagnosed infrequently. Most cervical fractures are caused by severe trauma such as vehicular impact, colliding while running, or falling from a height. ${ }^{1,2}$ Subsequently, trauma sufficient to cause these injuries is often fatal or leaves a poor prognosis prompting euthanasia., ${ }^{3,4}$ Reported treatment options include conservative management or surgery; controversy has historically surrounded which of these treatments will provide the best outcome. ${ }^{2,5,6}$ A more recent review of outcomes following spinal fracture and luxation in dogs and cats has documented surgical management with pins and polymethylmethacrylate (PMMA) cement which was associated with a significantly greater improvement in neurological status when compared with conservatively managed patients. ${ }^{3}$ Additionally, another recent publication assessing outcome following surgical intervention for cranial cervical vertebral fractures reported low mortality and complication rates. ${ }^{7}$

received

April 20, 2018

accepted after revision

May 3, 2018

Axial distraction of the cervical vertebral column can result in fracture fragments being pulled into alignment by the surrounding soft tissues. ${ }^{5-7}$ The vertebrae can subsequently be stabilized allowing fracture union. During fracture realignment and distraction, disruption of the traumatized tissues can result in severe, uncontrollable hemorrhage from damaged vascular tissues. Trauma to the cervical spine can disrupt local vascular structures such as the vertebral venous plexus. This severe hemorrhage can contribute to the high mortality rates reported with surgical intervention of spinal fractures. ${ }^{1,2}$ To the authors' knowledge, techniques to control such marked hemorrhage while fracture apposition and fixation are performed have not been reported and subsequently these scenarios provide a challenge to the surgeon and operating team.

This report describes two dogs that underwent surgical management of second cervical vertebrae (C2) fractures. We describe a surgical technique using pins and PMMA cement that allows hemorrhage limitation and simultaneous realignment

(c) 2018 Georg Thieme Verlag KG Stuttgart · New York
License terms

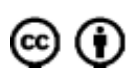

10.1055/s-0038-1660828. ISSN 2625-2325. 
and stabilization by distracting the vertebrae while cement is in a liquid phase. The short-, medium-, and long-term clinical outcomes are reported.

\section{Case Reports}

\section{Case 1}

A 1-year-old, Labrador Retriever dog was presented as an emergency following acute-onset tetraparesis earlier that day. The patient had been running out-of-sight through the forest, after which the gait abnormalities were apparent to the owner. The patient was ambulatory, had a low head carriage, and moderate ataxia was apparent with upper motor neuron lesions of all limbs. Signs of severe, diffuse pain were present on cervical spinal palpation. A C1-C5 spinal lesion was localized. A superficial skin wound was noted above the right orbit, suggestive of trauma following a collision.

The patient was premedicated with acepromazine (ACP; Novartis Animal Health UK Ltd, Camberley, UK; 0.02 mg/kg IM) and methadone hydrochloride (Martindale Pharmaceuticals, Romford, UK; $0.3 \mathrm{mg} / \mathrm{kg} \mathrm{IM}$ ), induced with propofol (Abbott Laboratories Ltd, Maidenhead, UK; $4 \mathrm{mg} / \mathrm{kg} \mathrm{IV),} \mathrm{and}$ maintained with isoflurane (Isoflo; Abbott Laboratories Ltd) in oxygen. Preoperative radiography (Elkin Medical Systems Inc, Santa Clara, California, United States) of the cervical spine (lateral) and computer tomography (CT; Siemens Somatom Emotion, Siemens AG Medical Solutions, Germany) images in multiple planar reconstructions demonstrated a moderately displaced, comminuted fracture of the vertebral body of C2 (-Fig. 1 ).

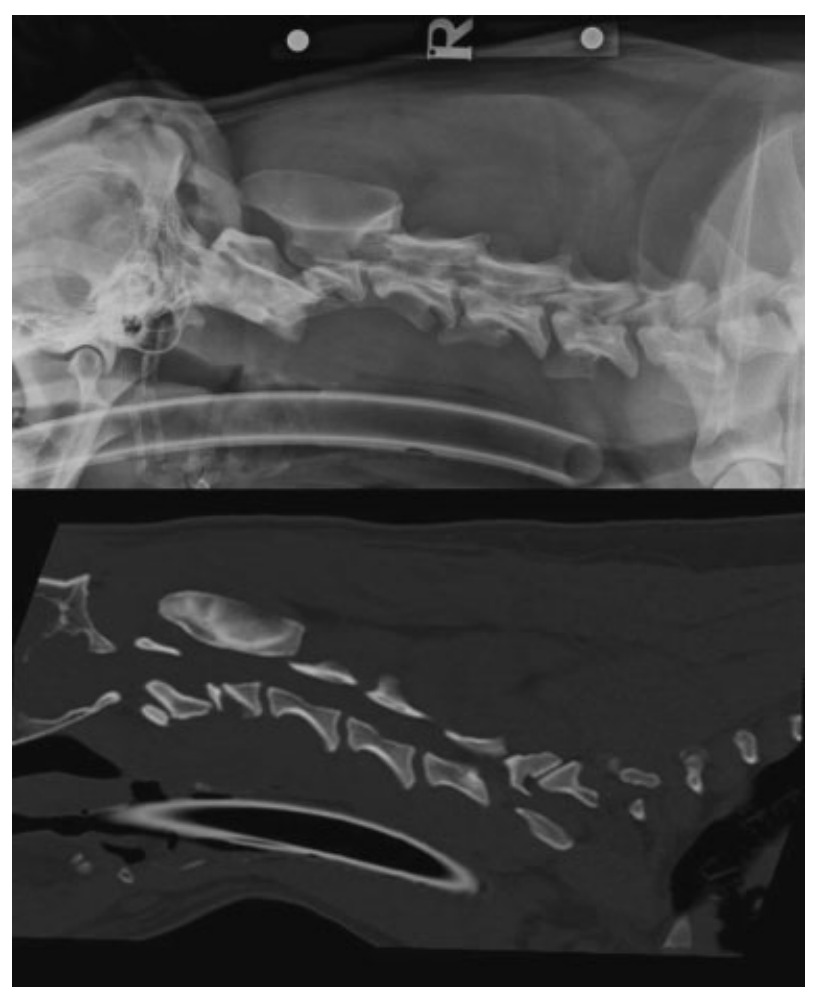

Fig. 1 Preoperative lateral radiograph and sagittal CT image of the cervical spine demonstrating displaced comminuted fracture of the C2 vertebra (case 1$)$.

\section{Surgery Report}

Cefuroxime (Zinacef: GlaxoSmithKline, Middlesex, UK; $22 \mathrm{mg} / \mathrm{kg} \mathrm{IV}$ ) was administered 30 minutes prior to the first incision and every 90 minutes for the duration of the procedure. Meloxicam (Metacam: Boehringer Ingelheim, Bracknell, UK; $0.2 \mathrm{mg} / \mathrm{kg} \mathrm{SC}$ ) was administered preoperatively.

A standard ventral midline approach was made to the cervical spine ${ }^{8}$ with retraction of the trachea, esophagus, and associated structures to the left. The longus colli muscles were divided along the midline and retracted to expose the ventral aspects of the $\mathrm{C} 1-\mathrm{C} 3$ vertebrae. Careful exploration and distraction of the fracture site demonstrated rupture and entrapment of a vertebral venous sinus within the fracture site, resulting in profuse hemorrhage during attempts to reduce the fracture.

Pins (INTERFACE Fixation Half-pins; IMEX, Longview, Texas, United States; $2.0 \mathrm{~mm}$ diameter) were embedded into the vertebral bodies of $\mathrm{C} 2$ (both cranial $[n=2]$ and caudal $[n=3$ ] to the fracture) and C3 $(n=2)$ and into the base of the wings of $\mathrm{C} 1$ bilaterally $(n=4)$. The four most cranial and caudal pins of the construct were left extruding further than the others and were bent to form a "hooked" end. Two pairs of Gelpi self-retaining retractors were preplaced between the hooked ends of the cranial and caudal pins (-Fig. 2). The Gelpi retractors were positioned so that they were raised above the spine, ensuring that they were not encased within the cement when it was later applied. Absorbable lyophilized bovine collagen fleece (Lyostypt; $B$. Braun Aesculap, Tuttlingen, Germany) was packed directly over the nonreduced fracture and around the occipital fossa. Following this, a large quantity of autogenous cancellous bone graft collected bilaterally from the proximal humeral metaphysis was overlaid onto the entire ventral surface of $\mathrm{C} 2$

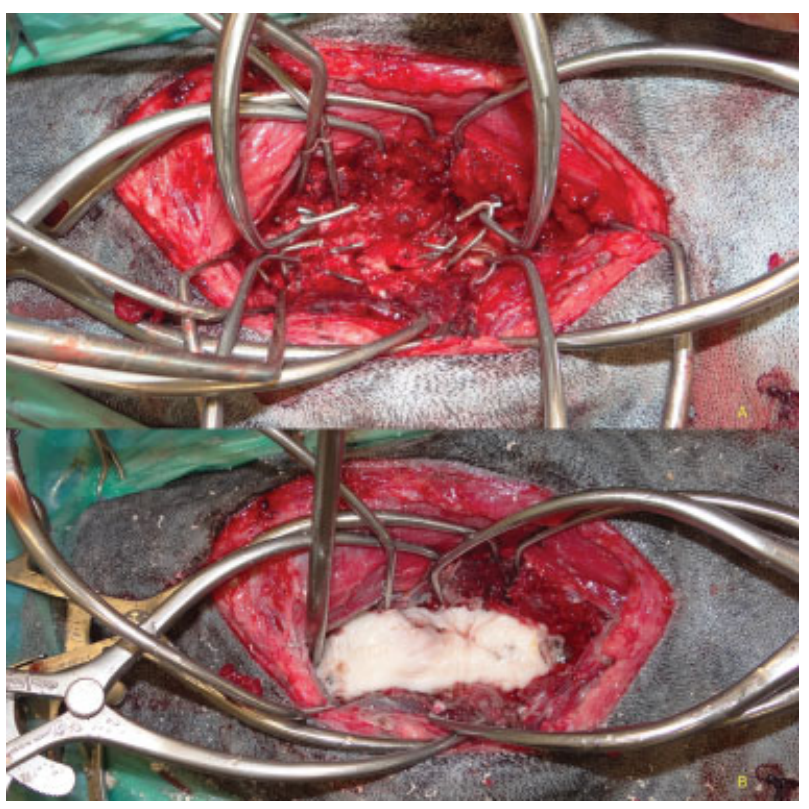

Fig. 2 Intraoperative photograph showing (A) pin and Gelpi retractor placement prior to graft and cement placement, and (B) after the cement bolus had cured and hooked pin tips used for distraction excised (case 1). Cranial is to the left of the photograph. 
and packed into the articular facets of $\mathrm{C} 1-\mathrm{C} 2$ following cartilage debridement (-Fig. 2).

Tobramycin-impregnated PMMA bone cement (Antibiotic Simplex P; Howmedica Osteonics, Limerick, Ireland) was subsequently prepared by hand mixing and poured onto the prepared bone graft bed and surrounding the pins. While still in liquid phase, axial distraction was applied using the preplaced Gelpi retractors. This served to limit the hemorrhage, with the collagen fleece, bone graft, and cement providing a physical barrier to ongoing hemorrhage outside the spinal canal.

After curing, the cement bolus and pin tips were contoured in situ with a high-speed burr and small pieces of cement at the periphery removed with rongeurs to avoid impingement on the occipital condyles or cervical soft tissues (-Fig. 2). Moistened gauze swabs were laid over the surrounding soft tissues to limit contamination with cement debris prior to burring. The surgical site was thoroughly lavaged. Soft-tissue closure in several layers was routine.

Postoperative radiography and CT confirmed appropriate implant placement and reasonable fracture reduction ( - Fig. 3 ).

\section{Postoperative Management}

The administration of methadone $(0.3 \mathrm{mg} / \mathrm{kg})$ was continued for 48 hours postsurgery; then the dose was reduced $(0.15 \mathrm{mg} / \mathrm{kg})$ for 24 hours and administered every 4 hours. Following this, buprenorphine (Vetergesic: Ceva Animal Health, Amersham, Buckinghamshire, UK; $0.02 \mathrm{mg} / \mathrm{kg}$ ) was administered every 8 hours for 24 hours. Oral meloxicam $(0.1 \mathrm{mg} / \mathrm{kg}$ ) was administered once daily for 4 weeks and oral cephalexin (Therios: Ceva Animal Health, Amersham, Buckinghamshire, UK; $22 \mathrm{mg} / \mathrm{kg}$ ) was continued twice daily for 10 days postoperatively.
There was no decline in neurological status following surgery and by 6 weeks postoperatively, the patient was considered to be clinically normal; radiographs and CT demonstrated no implant disruption and progression toward osseous union of the fracture. Radiography and CT at 12 weeks postoperatively demonstrated osseous union. As an incidental finding, two of the pins placed into the wings of C1 had broken, presumably as a result of incomplete intervertebral fusion between the $\mathrm{C} 1$ and $\mathrm{C} 2$ vertebrae. There was no evidence of pin migration. Exercise was restricted to controlled lead walks of increasing duration for the first 12 weeks using a harness. Follow-up examination 6 months after surgery documented a neurologically normal patient; radiographs and CT demonstrated no further implant disruption (-Fig. 4). A telephone interview with the owner 4 years following surgery revealed the patient remained free of clinical signs.

\section{Case 2}

A 2-year-old, Lurcher dog was presented as an emergency following acute-onset nonambulatory tetraparesis. The dog had collided its head with a tree that same day. There were reduced withdrawal reflexes affecting the thoracic limbs, and to a lesser extent the pelvic limbs. Voluntary motion was absent in the left thoracic limb, and reduced in the right thoracic and in both pelvic limbs. There were signs of severe neck pain present on palpation. A $\mathrm{C} 1-\mathrm{C} 5$ cervical spinal lesion was localized with concurrent spinal shock.

\section{Surgery Report}

The anesthetic technique was the same as for case 1 . Preoperative radiographs and CT images in multiple planar

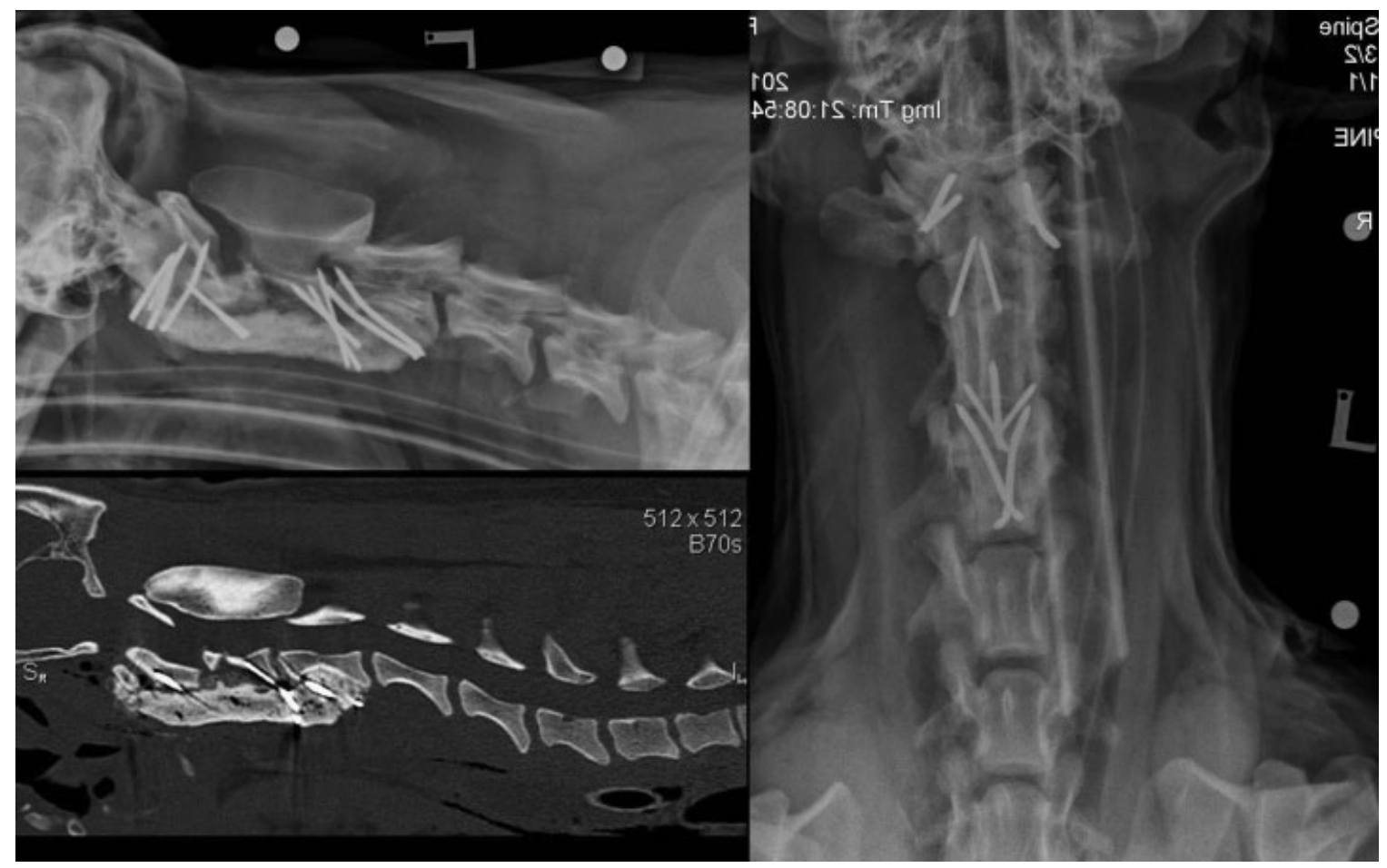

Fig. 3 Immediate postoperative lateral and ventrodorsal radiographs and sagittal CT image of the cervical spine demonstrating fracture reduction and implant position (case 1 ). 


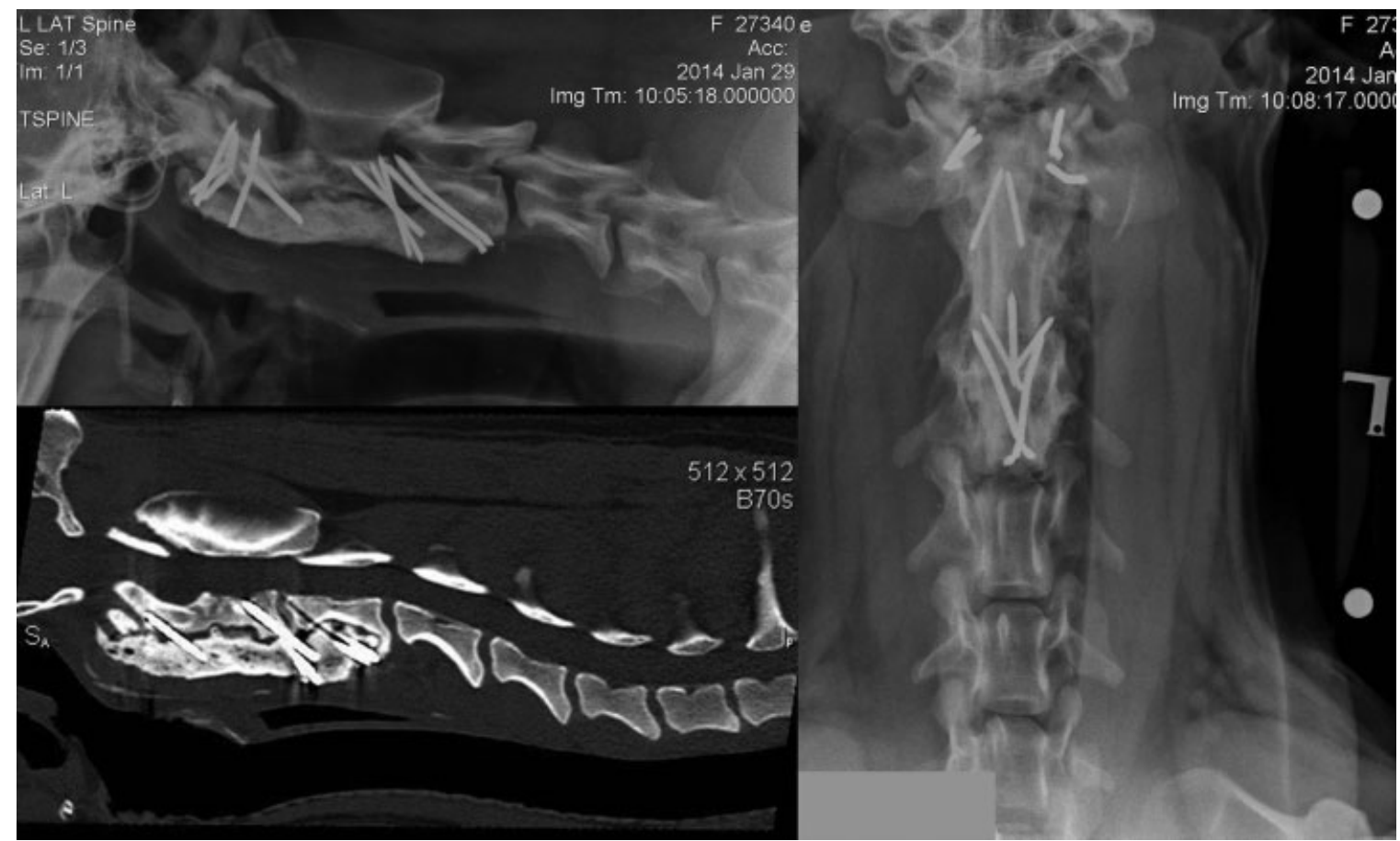

Fig. 4 Six months' postoperative lateral and ventrodorsal radiographs and sagittal CT image of the cervical spine demonstrating fracture union. Two pins in $\mathrm{C} 1$ were noted to have fractured as an incidental finding (case 1).

reconstruction demonstrated a displaced, comminuted fracture of the vertebral body of C2 (-Fig. 5 ).

The anesthesia protocol and surgical approach were the same as for case 1. Again in this patient, profuse hemorrhage occurred, filling the surgical field when attempts were made to

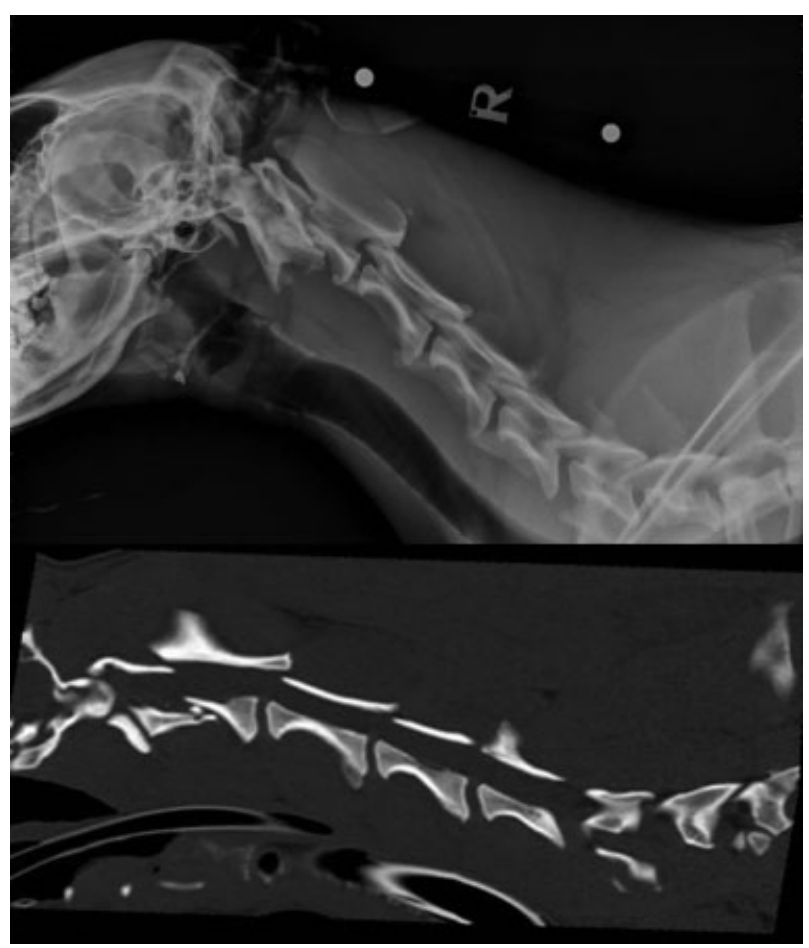

Fig. 5 Preoperative lateral radiograph and sagittal CT image of the cervical spine demonstrating displaced comminuted fracture of the C2 vertebra (case 2 ). reduce the fracture fragments, by applying traction to the vertebrae. Pins ( $2.0 \mathrm{~mm}$ diameter; IMEX, Longview; $n=7$ ) were inserted into $\mathrm{C} 1$, two of which passed through the cranial fragment of $\mathrm{C} 2$ and through the facet joints into $\mathrm{C} 1$. Pins $(n=3)$ were also embedded in the caudal vertebral body of C2 plus in the cranial $(n=3)$ and caudal $(n=2)$ body of C3 (-Fig. 6). Orthopaedic wire was passed around the pins

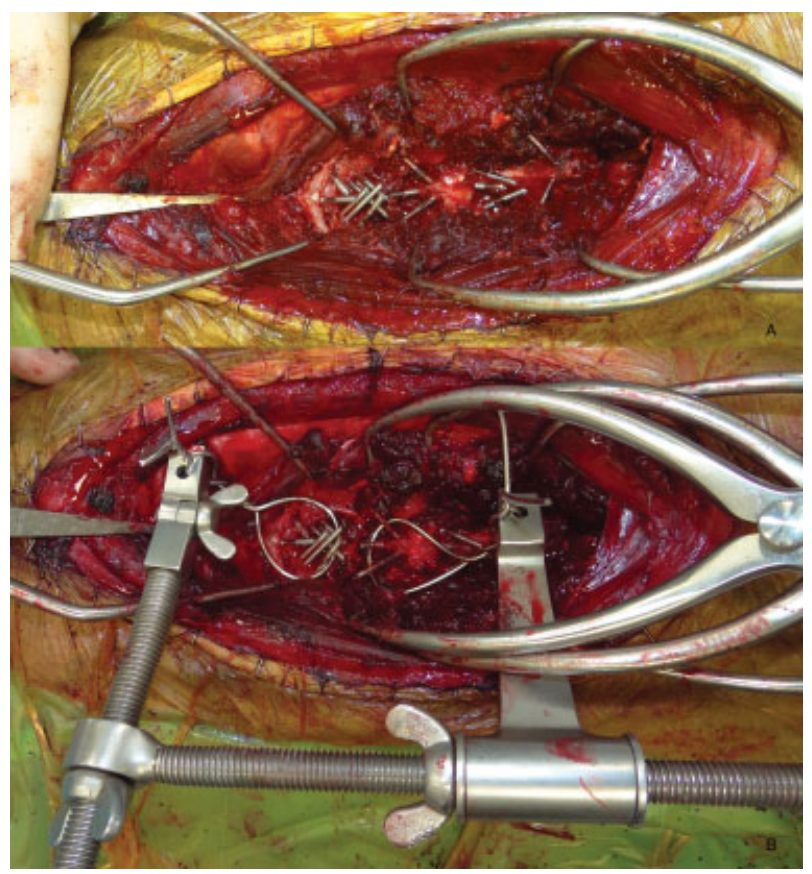

Fig. 6 Intraoperative photograph showing (A) pin placement, and (B) orthopaedic wire and retractor used for axial distraction (case 2). Cranial is to the left of the photograph. 
immediately cranial and caudal to the fracture site. These wires were then passed through a multidirectional adjustable jig (Orthopaedic Distractor Ref: 001500; Veterinary Instrumentation, Sheffield, UK; - Fig. 6). As described for case 1, absorbable collagen fleece (B. Braun Aesculap) and autogenous cancellous bone graft were placed over the fracture site. PMMA cement (Howmedica Osteonics) was then poured to enshroud the pins and wire. While the cement was solidifying, axial distraction was applied via the wires using the multidirectional jig. After curing, the orthopaedic wire protruding from the cement bolus was excised flush with the contour of the cement and closure was routine.

The immediate postoperative radiographs and CT images (-Fig. 7) confirmed satisfactory fracture alignment to reestablish the spinal canal.

\section{Postoperative Management}

The administration of methadone $(0.3 \mathrm{mg} / \mathrm{kg})$ was continued for 48 hours postsurgery and administered every 4 hours. Following this, buprenorphine $(0.02 \mathrm{mg} / \mathrm{kg})$ was administered every 8 hours for 24 hours. Oral meloxicam $(0.1 \mathrm{mg} / \mathrm{kg})$ was administered once daily for 4 weeks and oral gabapentin (10 mg/kg) was administered three times daily for 3 weeks. Oral cephalexin $(22 \mathrm{mg} / \mathrm{kg}$ ) was continued twice daily for 10 days postoperatively. There was no decline in neurological function following surgery. The patient was capable of ambulating unassisted 2 days following surgery.

Exercise was restricted to controlled lead walks of increasing duration for the first 12 weeks using a harness. At 6 weeks postsurgery, mild paresis remained; postural reactions of the pelvic limbs were mildly reduced, but the thoracic limbs were considered normal. Radiographs and CT demonstrated no implant disruption. There were not any signs of pain on palpation or manipulation of the cervical spine. Radiographs and CT at 12 weeks postoperatively demonstrated osseous union of the fracture ( $\mathbf{- F i g . 8}$ ) and the degree of ataxia had improved further, but not fully resolved. The patient was returned to the hospital 1 year later for assessment of a pelvic limb lameness due to coxofemoral osteoarthritis secondary to hip dysplasia. No signs of pain were present on palpation or manipulation of the cervical spine and ataxia had resolved. Radiographs and CT of the cervical spine revealed progressive fracture site remodeling and no implant-related complications. A telephone interview with the owner 3 years following surgery revealed the patient remained free of clinical signs.

\section{Discussion}

We report successful clinical outcomes after the use of a novel technique for reduction and stabilization of C2 vertebra fractures while concurrently limiting severe vertebral venous plexus hemorrhage in two dogs. Resolution of pain and improvement in neurological status occurred in both patients. The only complication included postoperative implant failure in one dog with no apparent clinical significance and did not require further treatment. These cases illustrate the application and successful outcomes for performing simultaneous fracture reduction and fixation while the cement is polymerizing in liquid phase to concurrently limit vertebral venous plexus hemorrhage in dogs.

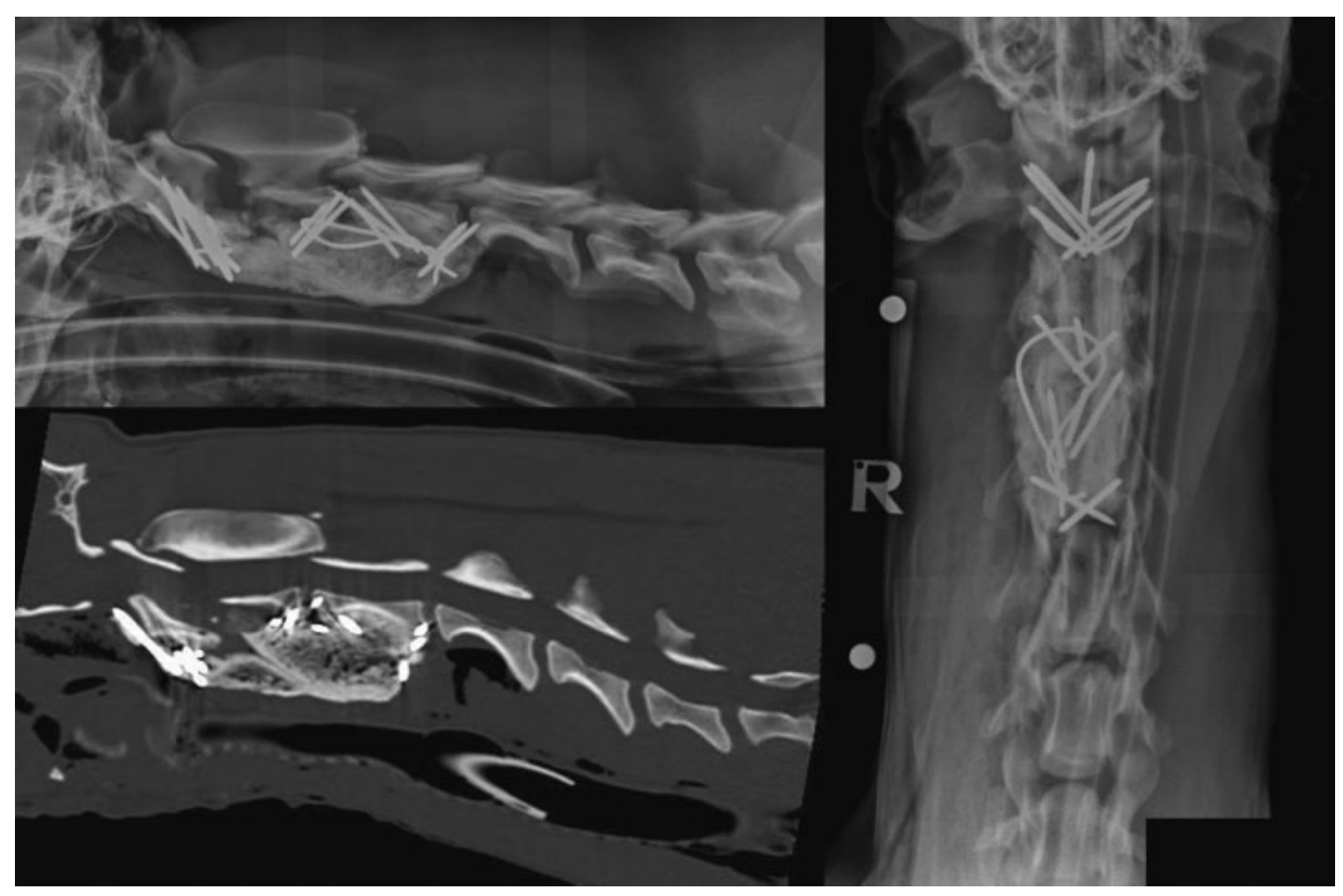

Fig. 7 Immediate postoperative lateral and ventrodorsal radiographs and sagittal CT image of the cervical spine demonstrating fracture reduction and implant position (case 2 ). 


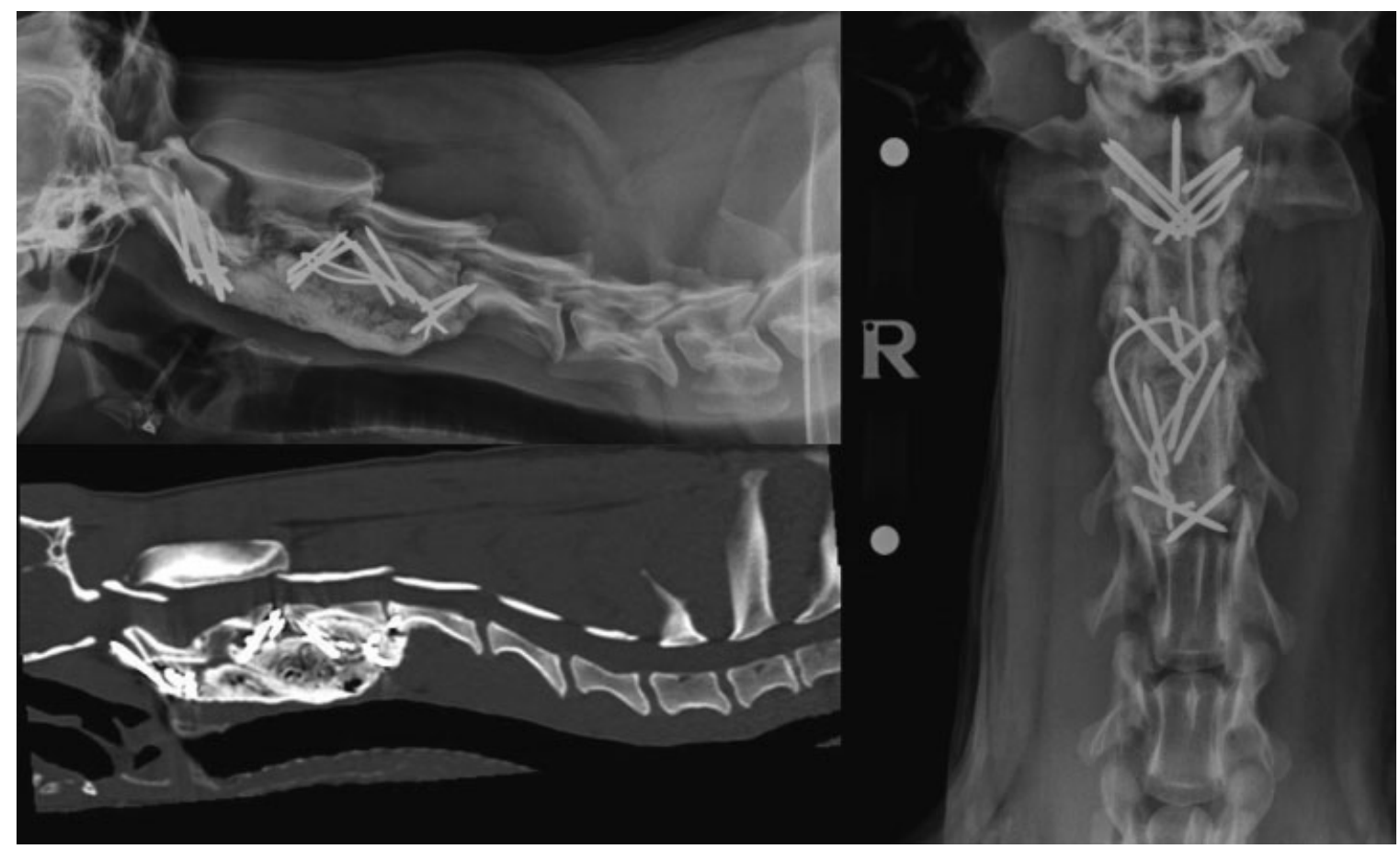

Fig. 8 Twelve weeks postoperative lateral and ventrodorsal radiographs and sagittal CT image of the cervical spine demonstrating fracture union (case 2).

The most frequently performed surgical technique for fractures of the cervical spinal vertebrae includes the use of ventrally applied pins (or screws) into the vertebrae with the ends enshrouded in PMMA cement. ${ }^{3,5-7}$ Alternatively reported surgical methods include ventrally ${ }^{9}$ or dorsally applied plates. ${ }^{5}$ Traditional techniques for fracture management involve reduction of the fractures using manipulation and instrumentation and subsequently application of implants to provide stability. For cervical vertebrae fractures and luxations, axial distraction can assist fracture reduction as the surrounding soft tissues pull the fragments back into alignment. ${ }^{7}$ While attempting distraction and reduction in these two patients, severe hemorrhage occurred from the vertebral venous plexus, stopping only when distraction was released and fracture fragments were allowed to return to their unaligned positions. Therefore, this technique had to be abandoned and an alternative solution applied.

We placed the retractors on the pins rather than on the patient's tissues; this has the advantage of elevating the retractors away from the ventral surface of the spine where the cement would be placed. Additionally, an advantage of placing the retractors on the pins is that no disruption or modification of the patient's spinal structures is required; this has been a previously reported technique to place retractors. ${ }^{7}$ The authors of this previous report ${ }^{7}$ described the use of disc fenestration to anchor the distractors and ex vivo studies in dogs have shown that cervical disc ventral fenestration can result in instability. ${ }^{10}$ However, a potential disadvantage of placing the distractors on the implants is causing them to deform or loosen during traction, which may negatively impact the overall construct strength. Indeed in case 1 , bending of the pins was observed on the postopera- tive imaging, but these pins were not the ones that subsequently broke. Therefore, we recommend that sufficient numbers of pins are placed so that the construct strength is not reliant solely on the pins which are used for distraction. Additionally, the use of orthopaedic wire looped around multiple pins, as was used in case 2, allows the distraction forces to be distributed, therefore reducing the impact on individual implants.

The vertebral venous plexus is an extensive vascular network that can become damaged following traumatic injury or iatrogenic disruption during surgery. Hemorrhage can be severe enough to result in death. Marked intraoperative hemorrhage can be difficult to control, as there is infrequently a single source of hemorrhage due to the network arrangement of the plexus. The cancellous bone graft placed over the ventral aspect of the vertebral column helps form a physical barrier to both reduce hemorrhage from the venous plexus and to protect the tissues of the vertebral column from infiltration and excessive heating from the cement. The bone graft has the potential to assist longer-term stability by forming an osseous bridge along the vertebral column, as demonstrated in -Figs. 4 and 8. However, the heat generated by the exothermic reaction of the curing cement could adversely affect the viability of cells within the graft.

Both patient outcomes were excellent with a resolution of pain and improvement in neurological status following surgery. The excellent clinical outcomes occurred despite two pins breaking in case 1 and two pins penetrating the $\mathrm{C} 3-\mathrm{C} 4$ intervertebral disc space in case 2 . Osseous union was achieved by 12 weeks for both patients as determined by CT images. Fracture healing can be challenging to assess from radiographs because of obscureness by the PMMA cement; ${ }^{7} \mathrm{CT}$ imaging can 
aid overcome this problem and is therefore recommended. Immediate postoperative CT imaging is also advised to ensure no fracture fragments or implants penetrate the spinal canal.

Both patients sustained fractures of $\mathrm{C} 2$. This is the most commonly reported fractured cervical vertebra in the veterinary literature with an incidence of $78 \%{ }^{1}$ and the vertebral body is the most commonly fractured portion of the vertebra occurring in $50 \%$ of cases. ${ }^{11}$ The high incidence of $\mathrm{C} 2$ fractures has been attributed to the lack of dorsal facet joints between $\mathrm{C} 1$ and $\mathrm{C} 2$, the poor dorsal ligamentous and tendinous support relative to the axial spine cranially and caudally, and the weak inertial properties of the cranial body of $\mathrm{C} 2$, particularly in axial bending. ${ }^{1}$ Preexisting atlantoaxial instability could result in a weak point within the cervical spine, but there was no evidence of dens hypoplasia in our patients.

Limitations to our technique are largely due to the potential problems following application of the liquid cement prior to distraction. The physical barrier created by the bone graft and cement prevent the surgeon from observing fracture alignment and distraction. The use of intraoperative fluoroscopy may help overcome this problem. Second, once the cement solidifies, adjustments cannot be made without prior removal of the cement. Therefore, we do not recommend that the technique we report is used as a first line of treatment but reserved for scenarios where excessive hemorrhage is impairing traditional fixation and all other strategies have been exhausted.

\section{Conclusion}

Severe hemorrhage from the vertebral venous plexus occurs infrequently during intraoperative repair of cervical fractures and luxations. However, if present it can prohibit the standard surgical approach of fracture reduction from axial traction followed by stabilization using internal implants. Preplacement of pins into the vertebrae followed by enshrouding of PMMA cement and application of axial traction while the cement is still in the liquid phase facilitated fracture alignment and fixation with limited hemorrhage. The clinical outcomes of two dogs that underwent this technique were excellent and could return to unrestricted, pain-free activities.
Conflict of Interest

None declared.

Funding

No grants or financial support received.

\section{Authors' Contributions}

James W. Guthrie and Noel Fitzpatrick contributed to the conception of study, study design, acquisition of data, and data analysis and interpretation. Both authors drafted and revised and approved the submitted manuscript.

\section{References}

1 Stone EC, Betts CW, Chambers JN. Cervical fractures in the dog: a literature and case review. J Am Anim Hosp Assoc 1979;12:463-471

2 Hawthorne JC, Blevins WE, Wallace LJ, Glickman N, Waters DJ. Cervical vertebral fractures in 56 dogs: a retrospective study. J Am Anim Hosp Assoc 1999;35(02):135-146

3 Bruce CW, Brisson BA, Gyselinck K. Spinal fracture and luxation in dogs and cats: a retrospective evaluation of 95 cases. Vet Comp Orthop Traumatol 2008;21(03):280-284

4 Bali MS, Lang J, Jaggy A, Spreng D, Doherr MG, Forterre F. Comparative study of vertebral fractures and luxations in dogs and cats. Vet Comp Orthop Traumatol 2009;22(01):47-53

5 Bruecker KA. Surgical treatment of spinal fractures, luxations, and subluxations. In: Bojrab MJ, ed. Current Techniques in Small Animal Surgery. Philadelphia, PA: Williams \& Wilkins; 1998:988-996

6 Sturges BK, LeCouteur RA. Vertebral fracture and luxations. In: Slatter D, ed. Textbook of Small Animal Surgery. Philadelphia, PA: Elsevier Science; 2003:1244-1261

7 Pike FS, Kumar MSA, Boudrieau RJ. Reduction and fixation of cranial cervical fracture/luxations using screws and polymethylmethacrylate (PMMA) cement: a distraction technique applied to the base of the skull in thirteen dogs. Vet Surg 2012;41(02):235-247

8 Piermattei DL, Johnson KA. Approach to cervical vertebrae and intervertebral disks 2-7 through a ventral incision. In: An Atlas of Surgical Approaches to Bones and Joints of the Dog and Cat. Philadelphia, PA: Saunders; 2004:56-61

9 Voss K, Steffen F, Montavon PM. Use of the ComPact UniLock System for ventral stabilization procedures of the cervical spine: a retrospective study. Vet Comp Orthop Traumatol 2006;19(01):21-28

10 Macy NB, Les CM, Stover SM, Kass PH. Effect of disk fenestration on sagittal kinematics of the canine C5-C6 intervertebral space. Vet Surg 1999;28(03):171-179

11 Feeny DA, Oliver JE. Blunt spinal trauma in the dog and cat: insight into radiographic lesions. J Am Anim Hosp Assoc 1980;16:885-890 